\title{
Analytical heat conduction model of annular composite fins
}

\author{
a) Chen Feng, Subramaniam Yugeswaran, Michael Gibbons, Sanjeev Chandra
}

b) Mechanical and Industrial Engineering, University of Toronto, Toronto, ON, Canada

\begin{abstract}
A two-dimensional analytical heat conduction model of an annular composite fin has been carried out. The composite fins composed of a porous polyethylene core, a square aluminum insert, and metallic zinc coating layers, was fabricated using wire-arc spraying technology. Analytical solutions of temperature distribution, energy dissipation and fin efficiency through the fins at natural convection condition have been proposed.
\end{abstract}

Keywords - composite fins; heat conduction; analytical model.

\section{INTRODUCTION}

Composite fins are used in industrial applications such as heat exchangers and heat sinks [1]. In such composite fins the thermal conductivities of the coating $\left(k_{c}\right)$ and substrate $\left(k_{s}\right)$ materials differ and the total heat transfer depends on the thermal conductivity ratio $\left(k_{d} / k_{s}\right)$. If this ratio is less than unity the coating material is less conductive than the substrate and insulates the fin, whereas a ratio greater than unity means that the coating enhances heat transfer.

Cortes [2] developed analytical solutions for heat transfer in composite fins with variable thickness varying the $k_{d} / k_{s}$ from 0.04 to 13.6 , which reflects combinations of metals commonly used in practical applications. Luna-Abad and Alhama [3] optimized the dimensions of composite rectangular fins while varying thermal conductivity ratios and convective heat transfer coefficients. Tu [4] derived analytical solutions for the efficiency of composite fins made of a metallic core and a coating with lower thermal conductivity.

In this paper we carry out an analytical model of heat conduction through an annular composite fin, consisting of a polymer substrate on which a thin metal coating was applied by wire arc spraying to enhance heat conduction. This model was developed assuming a two-dimensional conduction in the thicker polymer core and one-dimensional conduction in the thin coating layer because of their transverse thermal resistances. The model was validated by comparing predicted temperature variations with experimental measurements. The model was used to examine the effect of varying coating thickness and fin dimensions on heat dissipation and efficiency of the fins.

\section{FABRICATION AND EXPERIMENTAL SETUP}

\section{A. Fabrication of composite fins}

Wire-arc spraying was used in this study, in which an electric arc is struck between the tips of two continuously fed wires to melt them. A high velocity air jet strips the molten metal from the wires and creates a spray of droplets that impact on the substrate. This was used to coat a polyethylene sheet with zinc, which has a relatively low melting point $\left(420^{\circ} \mathrm{C}\right)$ and did not damage the substrate during coating.

A composite fin was made by laser cutting a $148 \mathrm{~mm}$ diameter disk from a $3.2 \mathrm{~mm}$ thick sheet of polyethylene. An aluminum block, $3.2 \mathrm{~mm}$ thick and $22 \mathrm{~mm}$ square was inserted into the center of the disk as shown in Fig. 1a and bonded to it using thermally conductive epoxy (DP270, 3M, St. Paul, USA). The relatively high thermal conductivity of the aluminum $(200 \mathrm{~W} / \mathrm{mK})$ ensured a uniform temperature that was used as a boundary condition in the analytical model. The model assumed radial symmetry and the fin was assumed to be an annulus with an internal radius of $11 \mathrm{~mm}$ and an outer radius of $74 \mathrm{~mm}$.

An electric wire arc spray system (ValuArc, Sulzer Metro Inc., Westbury, NY), mounted on a robot arm, was used to melt and spray high purity zinc wires (Catalog\#

1031592, Sulzer Metro Inc., Westbury, NY) onto the disk and form thin, dense zinc layers on both sides. The average coating thickness was kept at $400 \mu \mathrm{m}$. The thermal conductivity of the polyethylene $\left(k_{1}\right)$ was $0.3 \mathrm{~W} / \mathrm{mK}$ and that of the zinc $\left(k_{2}\right)$ was $60(\mathrm{~W} / \mathrm{mK})$. The final composite fin after coating is shown in Fig. 1b.

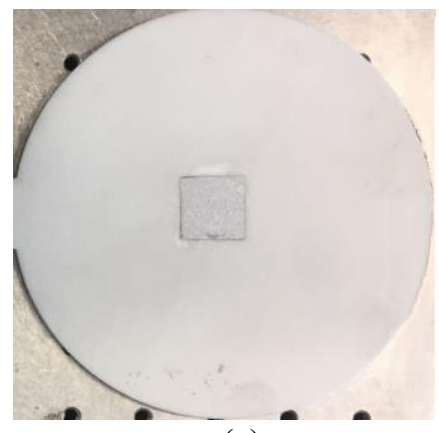

(a)

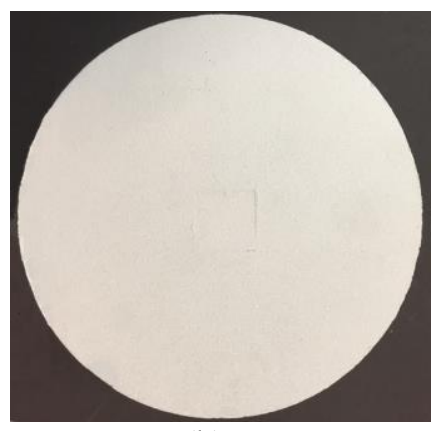

(b)
Figure 1. Pictures of the annular composite fin, (a) before spraying, and (b) after spraying. 


\section{B. Experimental setup}

The centre of the fin was heated using a flexible, silicone rubber heater, $23 \mathrm{~mm}$ square, (35765K126, McMaster Carr, Santa Fe Springs, California, USA) attached to the aluminum insert using thermal tape (TAPE-T404, Aavid Thermalloy, San Jose, California, USA). The other side of the heater was insulated using fiber glass insulation so that the heat lost from it was negligible. Moreover, the temperature variation on the fin surfaces was measured using an infra-red camera. The fin was mounted horizontally with an infrared camera (ThermaCAM SC 5000, FLIR, USA) pointing downwards to record the temperature of the upper surface.

The power supplied to the heart was varied to $1.6,2.5,4.0$ and $9.0 \mathrm{~W}$, and the fin allowed to come to steady state which took approximately $30 \mathrm{~min}$, and the temperature on the top fin surface recorded.

\section{ANALYTICAL MODELING}

The composite fin was circular with a rectangular crosssection, and the geometry can be simplified for purposes of modelling as shown in Fig. 2. The fin was constructed as a sandwich structure with a polymer core with thickness $2 H$, an outer radius $r_{0}$ and inner radius $x_{\mathbb{1}}$. Heat conduction in the twodimensional polymer core is governed by the following partial differential equation,

$$
\frac{\partial^{2} T_{1}}{\partial r^{2}}+\frac{1}{r} \frac{\partial T_{1}}{\partial r}+\frac{\partial^{2} T_{1}}{\partial y^{2}}=0
$$

Defining the following non-dimensional variable,

$$
r^{*}=\frac{\gamma-T_{i}}{L} ; y^{*}=\frac{y}{H} ; \quad \theta_{1}=\frac{T_{1}-T_{\text {mir }}}{T_{b}-T_{\text {mir }}} ; \quad \theta_{2}=\frac{T_{2}-T_{\text {mîr }}}{T_{b}-T_{\text {mîr }}}
$$

Where $\mathrm{L}$ is the characteristic length of the fin, defined as $r_{0}-r_{\mathrm{i}}, T_{\mathrm{b}}$ is the wall temperature of the aluminum insert and $T_{\text {air }}$ is the ambient air temperature, and $T_{1}$ and $T_{2}$ the temperature distributions in the polymer core and coating respectively. Substituting these dimensionless variable into equation (1) gives,

$$
\frac{\partial^{2} \theta_{1}}{\partial r^{42}}+\frac{L}{r} \frac{\partial \theta_{1}}{\partial y^{2}}+\frac{z^{2}}{H^{2}} \frac{\partial^{2} \theta_{1}}{\partial y^{22}}=0
$$

The following boundary conditions can be applied for the core,

$$
\begin{gathered}
r^{*}=0, \theta_{1}=1 ; \quad r^{*}=1, \frac{\partial \theta_{1}}{\partial y^{*}}=0 \\
y^{*}=0, \frac{\partial \theta_{1}}{\partial y^{*}}=0 \quad ; \quad y^{*}=1, \frac{\partial \theta_{1}}{\partial y^{*}}=\frac{k_{2}}{k_{1}} \frac{\partial \theta_{2}}{\partial y^{*}}
\end{gathered}
$$

Where $k_{1}$ and $k_{2}$ are thermal conductivity of the polymer core and the metallic coating layers, respectively. h, $T_{\text {air }}$

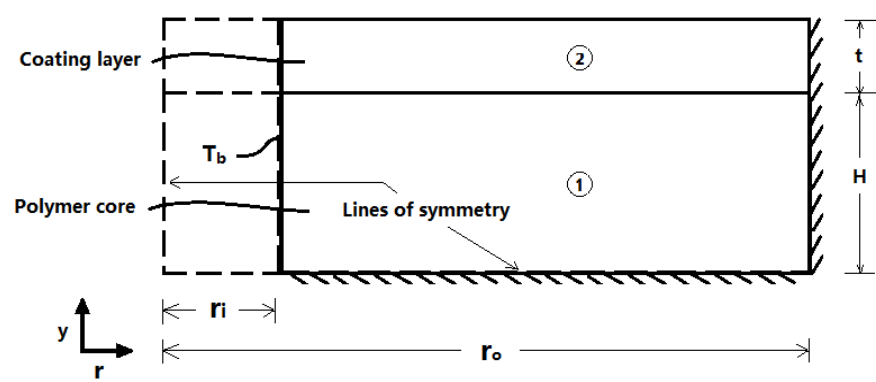

Figure 2. Analytical heat conduction model of the composite fin consisting of two domains.

Since heat transfer in the coating is assumed to onedimensional, with a temperature gradient only in the radial direction [5], an energy balance gives,

$k_{2} t\left(\frac{\partial^{2} T_{2}}{\partial r^{2}}+\frac{1}{r} \frac{\partial T_{2}}{\partial r}\right)-h\left(T_{2}-T_{\text {ăY }}\right)-\varepsilon \sigma\left(T_{2}^{4}-T_{\text {GiY }}^{4}\right)=\left.k_{1} \frac{\partial T_{1}}{\partial y}\right|_{y=H}$

The three terms on the left-hand side of equation (3) represent heat conduction, natural convection, and radiation in heat transfer, respectively. The term $T_{2}^{4}$ can be expanded in a Taylor series [6], and the new expression substitute into equation (3) gives,

$$
k_{2} t\left(\frac{\partial^{2} T_{2}}{\partial r^{2}}+\frac{1}{r} \frac{\partial T_{2}}{\partial r}\right)-h_{e}\left(T_{2}-T_{\text {aí }}\right)=\left.k_{1} \frac{\partial T_{1}}{\partial y}\right|_{y=H}
$$

Where $h_{e}=h+4 T_{\text {air }}{ }^{a} \varepsilon \sigma$ is an effective heat transfer coefficient that combines convection and radiation effects. Non-dimensionalizing equation (4) gives,

$$
k_{2} t\left(\frac{\partial^{2} \theta_{2}}{\partial y^{2}}+\frac{L}{r} \frac{\partial \theta_{2}}{\partial y^{m}}\right)-L^{2} h \theta_{2}-\left.\frac{L^{2}}{H} k_{1} \frac{\partial \theta_{1}}{\partial y^{y}}\right|_{y^{n}=1}=0
$$

With the boundary conditions,

$$
r^{*}=0, \theta_{2}=1 \quad ; \quad r^{*}=1, \frac{\partial \theta_{2}}{\partial \mathrm{r}^{\omega}}=0
$$

Using separation of variables by assuming that we can write $\theta_{1}$ as a product of two variable $R(r)$ and $Y(y)$ so that $\theta_{1}\left(r^{*}{ }^{*} y^{*}\right)=R\left(r^{*}\right) Y\left(y^{*}\right)$ and substituting in equation (2), gives two equations

$$
\begin{gathered}
Y^{m}+m^{2} Y=0 \\
R^{w}+\frac{L}{y} R^{s}-\frac{m^{2} L^{2}}{H^{2}} \mathrm{R}=0
\end{gathered}
$$

Where $m$ is an arbitrary separation constant. The general solutions of equation (6) and (7) are given [7],

$$
Y=c_{1} \cos \left(m y^{*}\right)+c_{2} \sin \left(m y^{*}\right)
$$




$$
R=c_{3} I_{0}\left(\frac{m L}{H} r^{*}+\frac{m}{H} \gamma_{\mathrm{i}}\right)+c_{4} K_{0}\left(\frac{m L}{H} r^{*}+\frac{m}{H} \gamma_{i}\right)
$$

Where $I_{n}(x)$ and $K_{n}(x)$ are the solutions to the modified Bessel differential function of the first and second kinds [8], [9]. At the interface between the core and the coating $(y=H)$ the temperatures in the core and coating are equal $\left(\theta_{1}\left(r^{*}, 1\right)=\right.$ $\left.\theta_{2}\left(r^{*}\right)\right)$. Substituting $\theta_{2}\left(r^{*}\right)=R\left(r^{*}\right) Y(1)$ into equation (5), we obtain,

$$
Y^{v}+\frac{H^{2} \hat{n}-k_{2} \operatorname{tm}^{2}}{k_{1} H} Y=0
$$

The boundary condition of $y^{*}=0, Y^{s}=0$ gives $c_{2}=0$ in equation (8). Substituting $Y=c_{1} \cos \left(m y^{*}\right)$ into equation (10), we obtain,

$$
\tan (m)=\frac{H^{2} \Re_{n}-k_{2} t m^{2}}{k_{1} H m}
$$

In our experiment $m$ is in a range of 0.028 to 0.032 , so we can use the approximation that for small $m, \tan (m) \approx m$ and introducing the fin parameter,

$$
M=\sqrt{\frac{\hbar}{k_{1} H+k_{2} t}}
$$

gives

$$
m=M H
$$

The coefficients $c_{3}$ and $c_{4}$ in equation (9) can be found by applying the boundary condition at $r^{*}=1_{a} \frac{\partial \theta_{1}}{\partial r^{*}}=0$. Then,

$$
c_{4}=\frac{I_{1}\left(M r_{0}\right)}{K_{1}\left(M r_{T_{Q}}\right)} c_{3}
$$

Combining the solutions for $R\left(r^{*}\right)$ and $Y\left(y^{*}\right)$ gives,

$\theta_{1}\left(r^{*}, y^{*}\right)=c_{5}\left[I_{0}\left(M L r^{*}+M r_{1}\right)+\right.$
$\left.\frac{I_{1}\left(M r_{0}\right) r_{0}\left(M L r^{*}+M r_{1}\right)}{N_{1}\left(M r_{0}\right)}\right] \cos \left(M H y^{*}\right)$

Where the only unknown parameter, $c_{5}$, can be obtained from the boundary condition that at $r^{*}=0, \theta_{1}=1$.

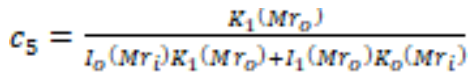

Then,

$$
\begin{aligned}
& \theta_{1}\left(r^{*}, y^{*}\right)=
\end{aligned}
$$

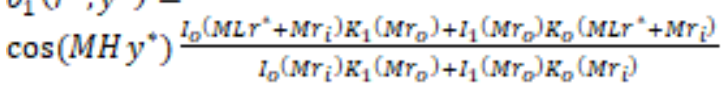

The radial temperatures of the fin core and coating must be the same at their interface so that $\theta_{2}(r)=\theta_{1}\left(r_{v} H\right)$. The radial variation of $\theta_{2}$ is, therefore,

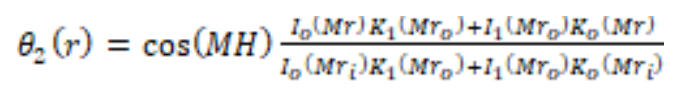

And

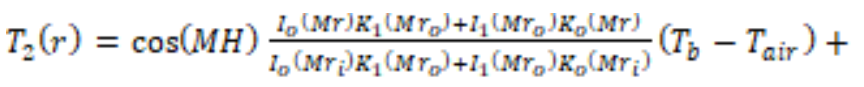
$T_{\text {air }}$

The heat conduction through the fin can be derived from,

$$
Q=\left.k A \frac{d T_{2}}{d y}\right|_{r=r_{i}}
$$

Where $A$ is the fin cross-section area at $r=\gamma_{\mathbb{1}}$. Evaluating equation (17) gives,

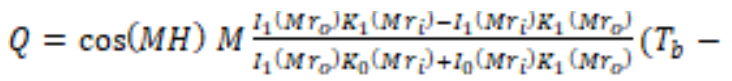

$$
\begin{aligned}
& \left.T_{\text {air }}\right) \cdot 2 \pi r_{1}^{(}\left(k_{1} H+k_{2} t\right)
\end{aligned}
$$

The fin efficiency can be defined as,

$$
\begin{aligned}
& \eta_{\text {fin }}=\frac{Q_{\text {fin }}}{Q_{\text {fĭn }}}
\end{aligned}
$$

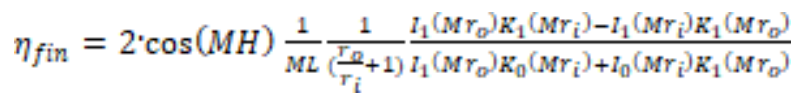

\section{RESULTS}

Fig. 3 shows both measured and calculated radial temperature variations on the top surface of the fin for four values of the heater power. The temperature is maximum closest to the heater and decreases radially. Increasing the heater power raises the temperature. Predictions from the analytical model agree well with experimental measurements. In the analytical model the natural convection coefficients are evaluated using the experimental correlation [10], which are $4.3,5.1,5.5$ and $6.6 \mathrm{~W} / \mathrm{m}^{2} \mathrm{~K}$ at the base temperature of 30,39 , 44 and $70{ }^{\circ} \mathrm{C}$, respectively, and the radiation heat transfer coefficient, $4 T_{\text {âI }}{ }^{3} \varepsilon \sigma_{s}$ is $5.1 \mathrm{~W} / \mathrm{m}^{2} \mathrm{~K}$. Radiation therefore plays a significant role in heat transfer from the fin. 


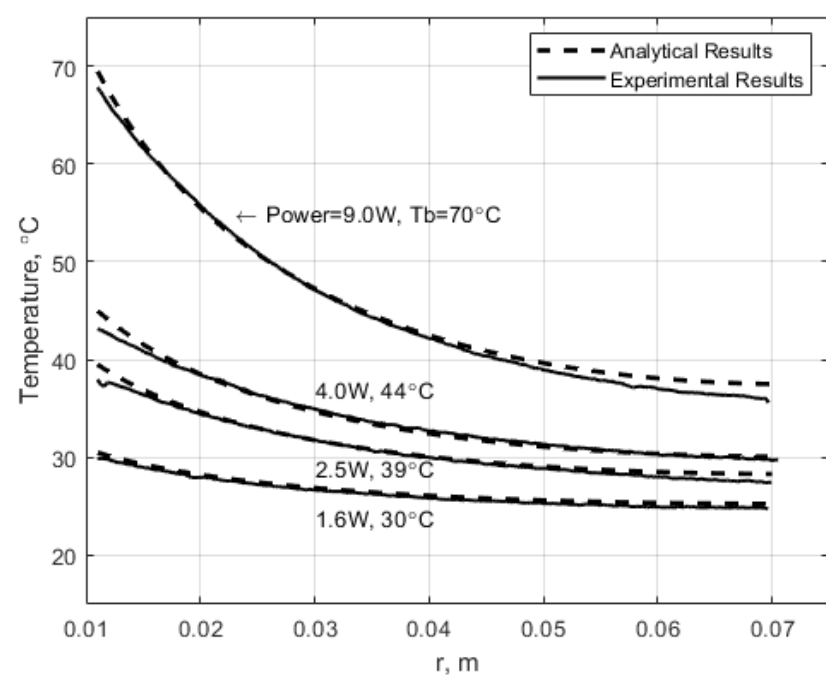

Figure 3. Analytical temperature curves of the composite annular fin (Equation 16) fits the experimental results at four heat input conditions, 1.6, $2.5,4.0$ and $9.0 \mathrm{~W}$.

Fig. 4 shows the variation of the base temperature of the fin, measured at the interface between the aluminium insert and the polymer fin, with the applied heater power. The base temperature increases with heater power in both the experimental and analytical results. The thermal resistance of natural convection and radiation is reciprocal to the effective heat transfer coefficient,

$$
R=\frac{1}{h_{e}}
$$

Known $h_{e}$ equals $h+4 T_{\text {air }}{ }^{a}$ हa and is a function of $R a$ and the ambinent temperature. $R a$ number depends on the fin temperature and increases with the base temperature. The natural convection coefficient increases with the power input, so the overall thermal resistance of natural convection and radiation reduces.

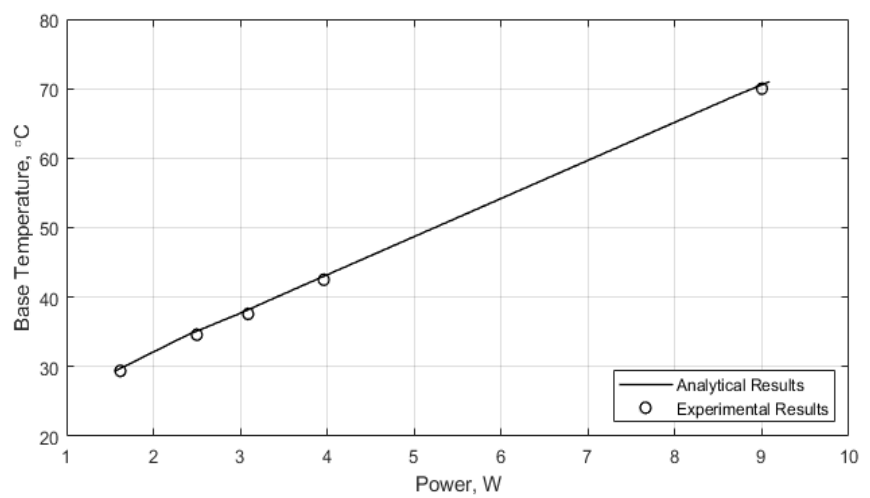

Figure 4. Analytical base temperature result of the composite annular fin (Equation 16) fits the experimental results at four heat input conditions, 1.6, $2.5,4.0$ and $9.0 \mathrm{~W}$.

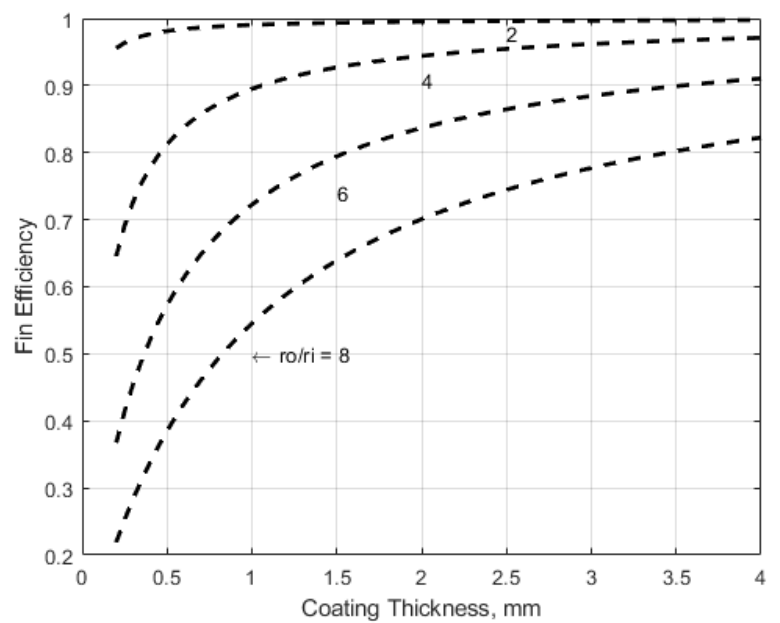

Figure 5. Fin efficiency of the composite annular fin (Equation 20) varies with the coating thickness at different ro/ri ratios.

Fig. 5 shows the variation in fin efficiency of annular composite (polymer/zinc) fins with coating thickness at four different $r_{o} / r_{i}$ ratios. Polymer core thickness, the inner fin radius $r_{i}$ and all thermal properties were assumed to be the same as those in our experiment. Fin efficiency increases with coating thickness in all cases because, as seen in Eqn 20, fin efficiency is a function of the fin parameter $M$ which decreases as coating thickness increases. For the annular fin that was tested in experiments, $r_{o} / r_{i}=6.7$ and the coating thickness was $0.4 \mathrm{~mm}$, giving a fin efficiency of approximately $50 \%$. Fin efficiency can be improved by reducing the fin radius since heat transfer decreases with distance from the center of the fin, so reducing $r_{0} / r_{i}$ or increasing the coating thickness would both improve efficiency.

\section{CONCLUSIONS}

An annular composite fin composed of a porous polyethylene core, a square aluminum insert, and metallic zinc coating layers, has been fabricated using wire-arc spraying technique. The analytical solutions of two-dimensional heat conduction model show an excellent prediction with the experimental results. The solutions indicate that the coating thickness has highly influence on the fin efficiency, and the fin efficiency can be accurately predicted by a single thermal length (ML), Eqn 20. The proper size of the fin can be determined by following the heat dissipation and fin efficiency curves of the fins in Figs 4 and 5. The solutions of this analytical model provide criteria in the annular composite fin design.

\section{REFERENCES}

[1] Wang R-T, Wang J-C. Analyzing the structural designs and thermal performance of nonmetal lighting devices of LED bulbs. International Journal of Heat and Mass Transfer. 
[2] Cortés C, Díez LI, Campo A. Efficiency of composite fins of variable thickness. International Journal of Heat and Mass Transfer. 2008;51(910):2153-66.

[3] Luna-Abad JP, Alhama F. Design and Optimization of Composite Rectangular Fins Using the Relative Inverse Thermal Admittance. Journal of Heat Transfer. 2013;135(8):084504.

[4] Tu P, Inaba H, Horibe A, Li Z, Haruki N. Fin Efficiency of an Annular Fin Composed of a Substrate Metallic Fin and a Coating Layer. Journal of Heat Transfer. 2006;128(8):851.

[5] Tu P, Inaba H, Horibe A, Li Z, Haruki N. Fin Efficiency of an Annular Fin Composed of a Substrate Metallic Fin and a Coating Layer. Journal of Heat Transfer. 2006;128(8):851.

[6] Weisstein EW. Taylor Series. MathWorld--A Wolfram Web Resource.
[7] Hille SLSGJEE. Calculus: One and Several Variables-Second order linear differential equations. 2006 10th Edition.

[8] Arfken G. Neumann Functions, Bessel Functions of the Second Kind, Nv(x). Mathematical Methods for Physicists. 1985:596-604.

[9] Arfken G. Bessel Functions of the First Kind, JV(x)" and "Orthogonality." Mathematical Methods for Physicists. 1985:573-91 and 91-96.

[10] P D, DeWitt. Fundamentals of heat and mass transfer. 2002. New York: J. Wiley. 